\title{
Analysis of Filter and Waveguide Effect Based on the MIM Nanodisk with a Metallic Block
}

\author{
Xiang Zhai, Yuanyuan Liu, Hongju Li, Rexidaiguli Wujiaihemaiti, \\ Yanhua Zhu, and Lingling Wang \\ Key Lab for Micro-Nano Physics and Technology, School of Physics and Electronics, Hunan University, \\ Changsha 410082, China \\ Correspondence should be addressed to Xiang Zhai; kele1110@hnu.edu.cn
}

Received 3 November 2014; Revised 12 January 2015; Accepted 12 January 2015

Academic Editor: Christian Brosseau

Copyright (C) 2015 Xiang Zhai et al. This is an open access article distributed under the Creative Commons Attribution License, which permits unrestricted use, distribution, and reproduction in any medium, provided the original work is properly cited.

\begin{abstract}
A novel and meaningful plasmonic filter based on the metal-insulator-metal (MIM) waveguides directly connected to a nanocavity with a metallic block is proposed and demonstrated numerically. By the effective index method and the resonant theory of diskshaped nanocavity, we reveal that the resonant wavelengths can be simply tuned by adjusting the height of the block, which is in accordance with the results calculated by finite-difference time-domain (FDTD) simulations. We also rotate the metallic block to achieve two resonant modes. One mode shows a blue shift, and the other mode shows a red shift. It is shown that the proposed structure performs as a bend waveguide not a filter when the width of the block increases to hundreds of nanometers. The proposed structure will have significant potential applications in nanointegrated circuits for optical filtering and processing.
\end{abstract}

\section{Introduction}

Surface plasmon polaritons (SPPs) are deriving from coupling of electromagnetic waves with oscillations of free electrons in a metal and supported at the insulator/metal interface [1]. Since SPPs can be confined in a subwavelengthscale area beyond the diffraction limit, they are regarded as information carriers for highly integrated photonic circuits, such as particles [2, 3], grooves [4-6], cavities [7-11], and apertures $[12,13]$. In addition to the merit of miniaturization for these devices, their unique characteristics can satisfy various applications. Take the basic waveguide, for instance, directional couplers and splitters, and even modulators utilizing electro/thermoeffect $[14,15]$. Typical plasmonic waveguides are the insulator-metal-insulator (IMI) waveguides and the metal-insulator-metal (MIM) waveguides [5, 16-21]. It is well known that the IMI waveguide exhibits less propagation loss, leading to longer propagation length than the MIM waveguide [16]. On the other hand, from a point of confining light, since the skin depth of SPPs fields into the dielectric medium is much longer than that into the metallic medium at the interfaces [19], the MIM waveguide is better than the
IMI waveguide. Thus, the MIM waveguide is more suitable for high optical integration.

Specifically, a fine filter-like behavior was achieved in a Bragg reflector alternatively stacking MIM waveguides with two kinds of dielectrics [22]. For the sake of simplifying the fabrication of Bragg reflectors and decreasing the propagation length for SPPs, some simple plasmonic filters have been proposed. For example, the proposed structure based on MIM waveguides with a nanodisk resonator acts as a band-pass filter [23]. It has also been reported that the one-end-sealed MIM waveguide reveals fine filtering effect [24]. Subsequently, unidirectional excitation of SPPs in Tshaped waveguide with nanodisk resonator has been analyzed theoretically and numerically [25]. More recently, comblike filters have been demonstrated [26]. These previous structures merely perform as filters. Thus, the investigation and design of a structure based on the MIM waveguides functioning not only as a filter but also as a bend waveguide are highly desired for various applications.

In this paper, we propose a novel structure based on the MIM waveguides directly connected to a nanodisk resonator with a metal-block core. In order to make our proposed 


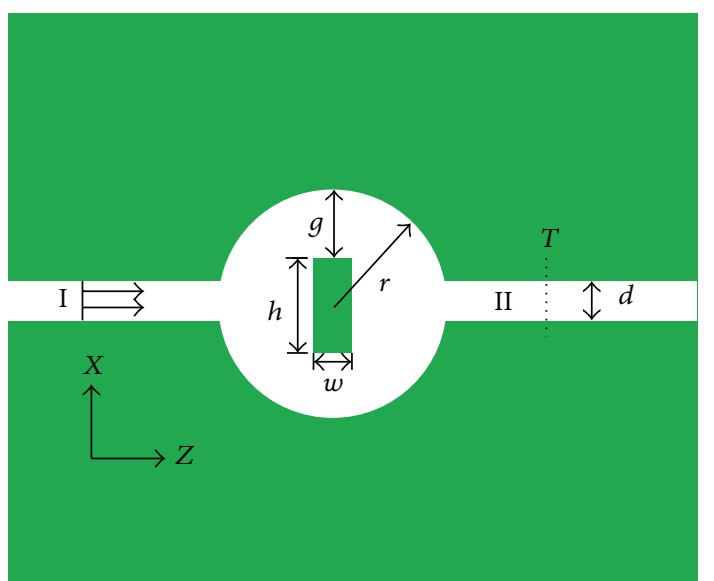

Silver

Air

Figure 1: Schematic diagram of the proposed plasmonic filter. $r$ : radius of nanodisk resonator, $h$ : height of metallic block, $w$ : width of metallic block, $g$ : width of Ag-air-Ag waveguide in the core of nanocavity, and $d$ : metal slit width.

structure be easily seized, we discuss the structure without the metal-block core at the beginning. The difference between simulation and theoretical results is related to the nanocavity not being sealed. Then we put the metallic block into the nanocavity. With the effective index method (EIM) and the resonant mode theory (RMT), we examine wavelength shifts of the resonant modes, which are also validated by finitedifference time-domain (FDTD) [27] method. Another result obtained by FDTD simulations reveals that regular shifts of resonant wavelengths can be manipulated by the block's rotation degree. Finally, when the width of the block changes from tens of nanometers to hundreds of nanometers, the proposed structure performs as a bend waveguide, not a filter.

\section{Model Creation and Theories}

Figure 1 illustrates the designed plasmonic filter which consists of two parts: two straight MIM waveguides (I, II) and a nanodisk cavity with a metal-block core. The two straight waveguides are connected to the cavity on the opposite sides, and the metallic block is located at the center of the cavity perpendicular to the straight waveguides. The width of straight waveguides $d$ is $50 \mathrm{~nm}$, and radius of nanodisk $r$ is $200 \mathrm{~nm}$. The height and width of the metallic block are labeled as $h$ and $w$, respectively, and the distance between the top of the block and the disk is $g$. The medium (the white areas) filled in the disk cavity and two straight waveguides is assumed as air with the refractive index being $1\left(n_{d}=1\right)$. The metal (the green area) is silver, whose complex relative permittivity modeled by Drude equation can be expressed as follows [4]:

$$
\varepsilon_{m}(w)=\varepsilon_{\infty}-\frac{w_{p}^{2}}{w(w+i \gamma)},
$$

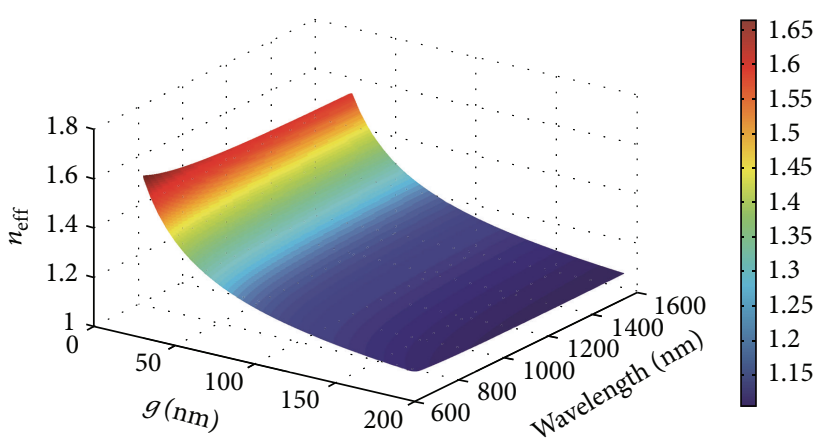

FIGURE 2: Influence of the width of the waveguide in the core of the nanocavity and wavelength on $n_{\text {eff }}$.

where $\varepsilon_{\infty}$ represents the dielectric constant at infinite angular frequency with a value of $3.7, w$ is the angular frequency of incident light, $w_{p}\left(1.38 \times 10^{16} \mathrm{rad} \mathrm{s}^{-1}\right)$ stands for the bulk plasma frequency, and $\gamma\left(2.73 \times 10^{13} \mathrm{rad} \mathrm{s}^{-1}\right)$ means the damping frequency of the electron's oscillation [28].

In this paper, we employ our 2D-FDTD program code to simulate the interaction between metal and incident waves. In our calculation, the current density is induced into Maxwell's equations since the real part of the permittivity of metal is negative. The grids are terminated by ten-cellthick convolutional perfectly matched layer (i.e., CPML). The spatial mesh cells are set to $\Delta x=\Delta z=\Delta s=5 \mathrm{~nm}$, and the time step and the total number of time steps are taken as $\Delta t=\Delta s / 2 c=8.33 \times 10^{-18} \mathrm{~s}$ and $2 \times 10^{4}$, respectively. The small size of cells and large total number of time steps ensure that the values of electromagnetic field and current density in spatial grids are convergent and stable. The fundamental TM mode of the MIM waveguide is excited by a plane wave source whose wavelength ranges from $600 \mathrm{~nm}$ to $1550 \mathrm{~nm}$. One frequency-domain field and power monitor is set to detect the transmission power $P$.

According to the Maxwell equations and boundary conditions, the effective refractive index of SPPs $\left(n_{\text {eff }}\right)$ in the metal-insulator-metal (MIM) structure can be obtained as follows [17]:

$$
\begin{gathered}
k_{d} \varepsilon_{m} \tanh \left(\frac{k_{d} d}{2}\right)+\varepsilon_{d} k_{m}=0, \\
k_{d, m}=\sqrt{\beta_{\mathrm{SPP}}^{2}-\varepsilon_{d, m} k_{0}^{2}}, \\
n_{\mathrm{eff}}=\frac{\beta_{\mathrm{SPP}}}{k_{0}},
\end{gathered}
$$

where $\varepsilon_{d}$ stands for the dielectric constant of the insulator and $\varepsilon_{m}$ is the dielectric constant of the metal, which can be obtained from (1). $k_{d}$ and $k_{m}$ are the transverse propagation constants of dielectric and metal, respectively. $k_{0}=2 \pi / \lambda$ is the free space wave vector of the excitation light. Figure 2 reveals that $n_{\text {eff }}$ relies on the width of the Ag-air-Ag waveguide in the core of the nanocavity $g$ and the wavelength $\lambda . n_{\text {eff }}$ declines with the increase of $g$ at the same wavelength, while it will be of no alteration by increasing the incident 


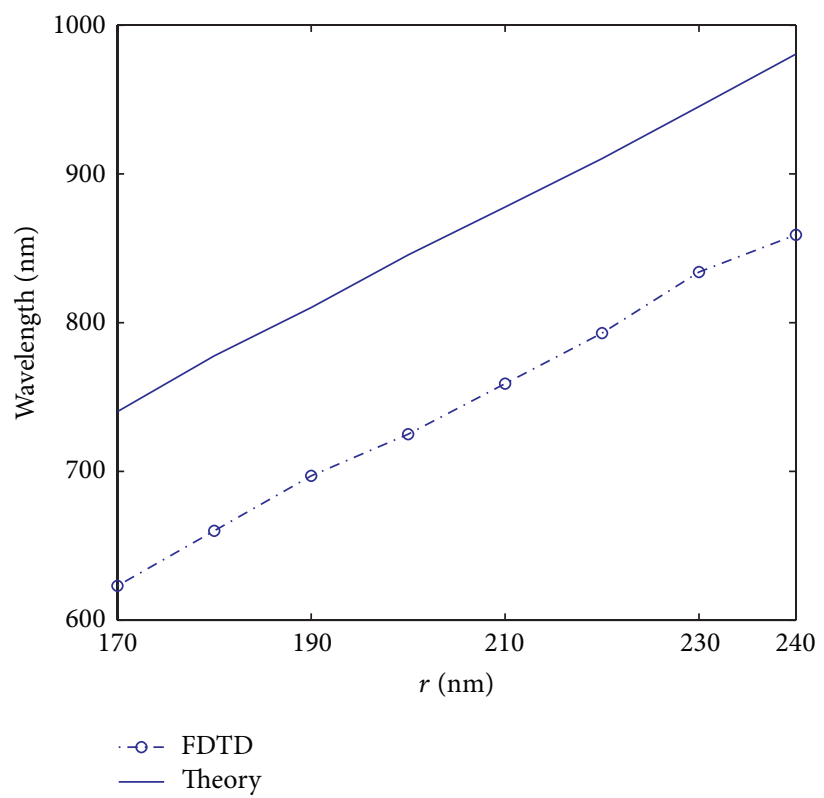

FIGURE 3: Resonant wavelength with respect to the radius of the cavity. The dot-dashed line is simulated from FDTD, and the solid line is calculated from (5).

wavelength with $g$ fixed. SPPs can propagate through the proposed structure when a stable standing wave is built up constructively in the cavity. In this case, the following resonant condition should be satisfied [29]:

$$
\Delta \psi=m \cdot 2 \pi
$$

where positive integer $m$ is the number of antinodes of the standing SPPs wave. $\Delta \psi$ is the phase delay per round-trip in the cavity, and we have got $\Delta \psi=2 \pi / \lambda \int_{0}^{L} n_{\text {eff }} d L$. So we can get the resonant wavelength:

$$
\lambda_{m}=\frac{\int_{0}^{L} n_{\mathrm{eff}} d L}{m}
$$

where $\lambda$ is incident wavelength and $L$ is the length per round trip. The resonant wavelength $\lambda_{m}$ increases with the increase of effective index $n_{\text {eff }} n_{\text {eff }}$ increases with the decline of $g$ (i.e., the increase of $h$ ) at the same wavelength from Figure 2. Therefore, $\lambda_{m}$ increases with the block's height increasing.

\section{Simulation Results and Discussion}

To start with, the transmission properties of the structure without a metal-block core inside the cavity are studied to be compared with the proposed structure. Such a structure presents a filter-like behavior. The resonant wavelength of the ideal disk-shaped nanocavity $[30,31]$ can be obtained by the following equation:

$$
k_{d} \frac{H_{n}^{(1) \prime}\left(k_{m} r\right)}{H_{n}^{(1)}\left(k_{m} r\right)}=k_{m} \frac{J_{n}^{\prime}\left(k_{d} r\right)}{J_{n}\left(k_{d} r\right)},
$$

where $r$ is the radius of the nanocavity. $J_{n}$ and $J_{n}^{\prime}$ are the first kind Bessel function with the order $n$ and its derivative. $H_{n}^{(1)}$ and $H_{n}^{(1) \prime}$ are the first kind Hankel function with the order $n$ and its derivative, respectively. Figure 3 reveals that the wavelength shift of the resonant mode has approximately linear relation with the radius of nanocavity from both (5) and FDTD result. The unideal nanocavity in our proposed structure (two edges in the middle connected to two waveguides and then not being sealed) has its effective radius. The actual radius not effective radius is taken into (5), which leads to the high discrepancy up to $100 \mathrm{~nm}$ between simulation and theoretical results.

Next, we put a metallic block into the nanodisk. We investigate the influence of the height of the block on the resonant wavelengths firstly by using the FDTD method and then by using the RMT. The height of the block $h$ is variable and the width $w$ is set as $50 \mathrm{~nm}$, while other parameters are fixed as above. Figure 4(a) shows the transmission spectra with the different heights of the block. From Figure 4(b) we can find that the resonant wavelengths tend to exhibit a red shift with the increase of the block's height, which is in accordance with (2)-(4). Magnetic-field $\left|H_{z}\right|$ distributions at $\lambda=728 \mathrm{~nm}$ with $h=0, \lambda=823 \mathrm{~nm}$ with $h=$ $100 \mathrm{~nm}$, and $\lambda=1066 \mathrm{~nm}$ with $h=200 \mathrm{~nm}$ are depicted in Figures 4(c)-4(e). We observe that the excitations of resonant modes emerge in the nanocavity under the three cases where the incident light can pass the MIM plasmonic structure. According to above simulations and analysis, it is obvious that we can achieve a fine filter-like behavior by tuning the height of the metallic block.

Another parameter $\theta$, which stands for the block's rotation degree, plays an important role in modulating the resonant wavelength. The rotation of the block leads to 


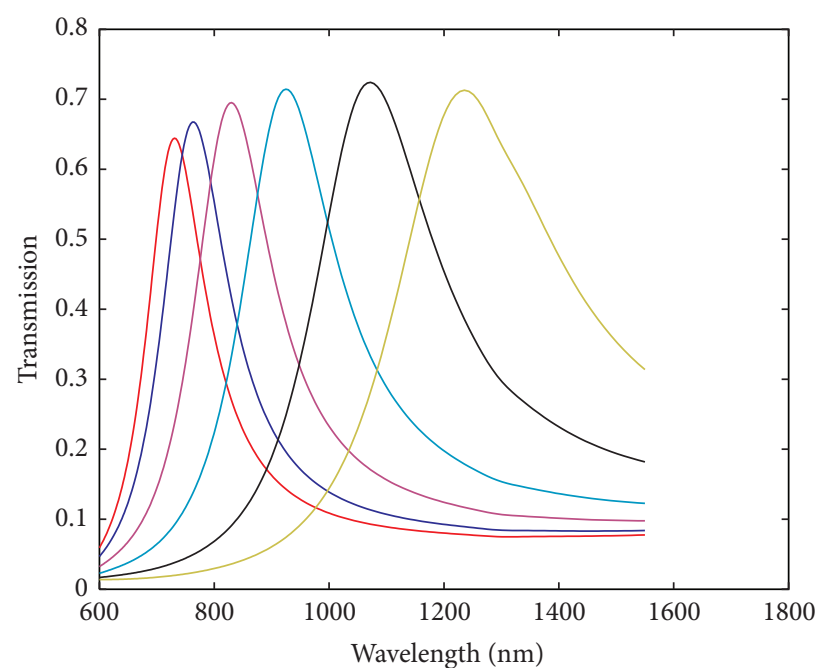

$\begin{array}{rlrl}h & =0 \mathrm{~nm} \\ h & =50 \mathrm{~nm} & -h & =150 \mathrm{~nm} \\ h & =100 \mathrm{~nm} & -h & =200 \mathrm{~nm} \\ h & =250 \mathrm{~nm}\end{array}$

(a)

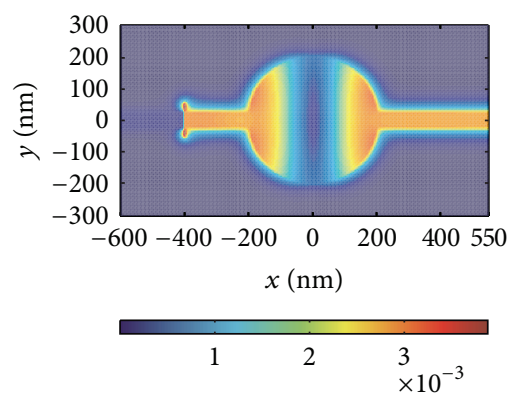

(c)

(d)

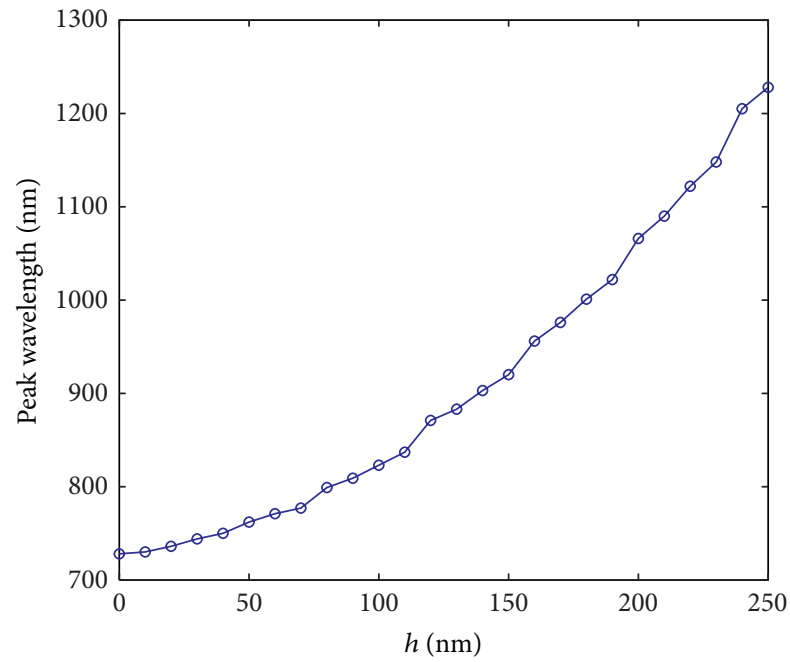

(b)

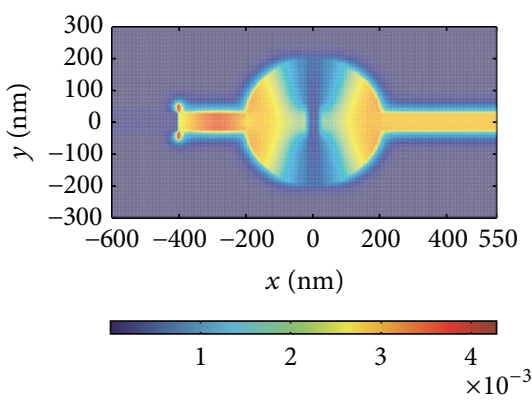

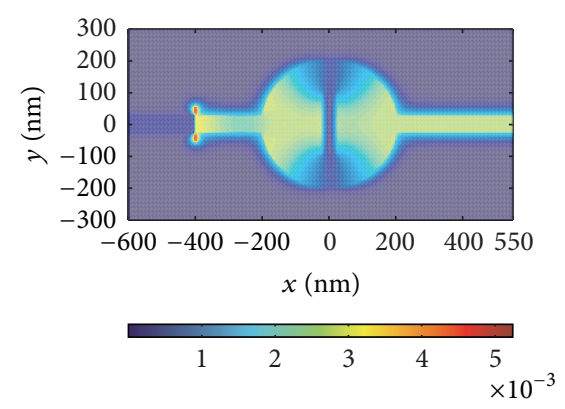

(e)

Figure 4: (a) Transmission response with respect to different heights of the block ( $w=50 \mathrm{~nm}$ ). (b) Resonant wavelength with respect to the height of the block. Contour profiles of field $\left|H_{z}\right|$ at (c) $\lambda=728 \mathrm{~nm}$ with $h=0 \mathrm{~nm}$, (d) $\lambda=823 \mathrm{~nm}$ with $h=100 \mathrm{~nm}$, and (e) $\lambda=1066 \mathrm{~nm}$ with $h=200 \mathrm{~nm}$.

a displacement of the dielectric core, which makes the direction of magnetic oscillation variable. In addition, the change of the direction of magnetic oscillation makes $g$ different, affecting $n_{\text {eff }}$ directly as shown in Figure 2 . Hence, it is clear that the effective index changes when the symmetry of the structure is destroyed by modulating the block's rotation degree $\theta$. The effective index $n_{\text {eff }}$ is an important factor influencing resonant wavelength $\lambda_{m}$ as shown in (4). Thus, we can manipulate the resonant wavelengths by changing the value of the block's rotation degree $\theta$, which is verified by FDTD simulations. The structural parameters are set as $w=50 \mathrm{~nm}$ and $h=200 \mathrm{~nm}$. Then we start to rotate the block counterclockwise. Figure 5(a) shows the transmission response corresponding to $\theta=0^{\circ}, 22.5^{\circ}, 45^{\circ}, 67.5^{\circ}$, and $90^{\circ}$. There is only one transmission peak at $\lambda=1066 \mathrm{~nm}$ when $\theta$ is zero. When the rotation degree is $90^{\circ}>\theta>0^{\circ}$, two transmission peaks appear around $\lambda=820 \mathrm{~nm}$ and $\lambda=$ $1096 \mathrm{~nm}$, which are called mode 2 and mode 1, respectively. With increase of rotation degree $\theta$, mode 1 exhibits a slight red shift, while mode 2 presents a blue shift (as shown in Figure 5(b)), leading to the wavelength gap between two modes getting larger. When $\theta$ is the value of $90^{\circ}$, only one transmission peak is observed at $\lambda=782 \mathrm{~nm}$. Furthermore, Figures 5(c)-5(e) depict the field distributions of $\left|H_{z}\right|$ of transmission peaks at $\lambda_{1}=820 \mathrm{~nm}$ and $\lambda_{2}=1096 \mathrm{~nm}$, and transmission valley at $\lambda_{3}=980 \mathrm{~nm}$ when $\theta$ is the value of $45^{\circ}$, which match Figure 5(a) perfectly. Therefore, one can choose special rotation degree to design the filter with demanded channel.

Finally, we find that the proposed structure changes from a filter to a waveguide under the special parameters. When we set the width of the block to be $250 \mathrm{~nm}$ and change the height from $210 \mathrm{~nm}$ to $250 \mathrm{~nm}$, the structure performs as a bend waveguide, not as a filter. In Figure 6(a), we show the calculated transmission as a function of wavelength. We can see that the loss is about $10 \%$ at longer wavelength. The critical wavelength $\lambda_{C}$ regarded as the original wavelength of the waveguide under which the transmission coefficient 


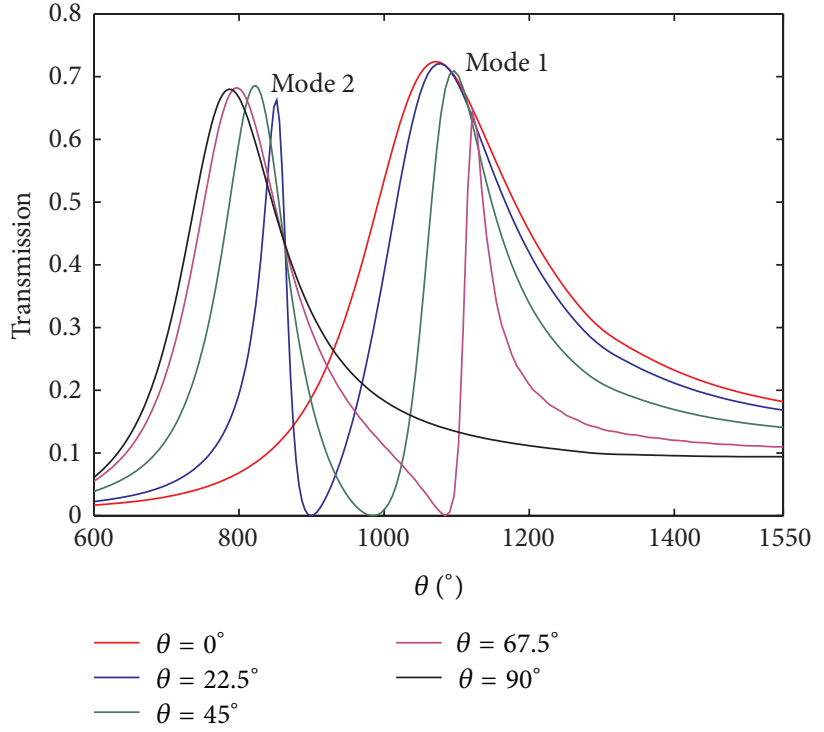

(a)

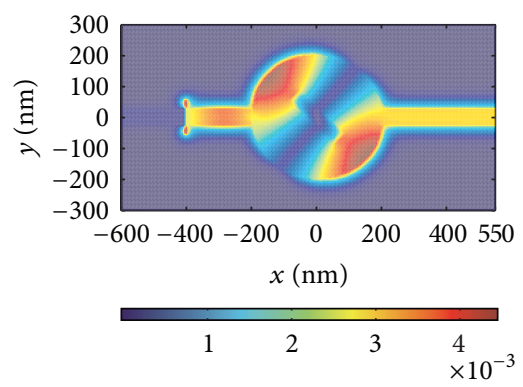

(c)

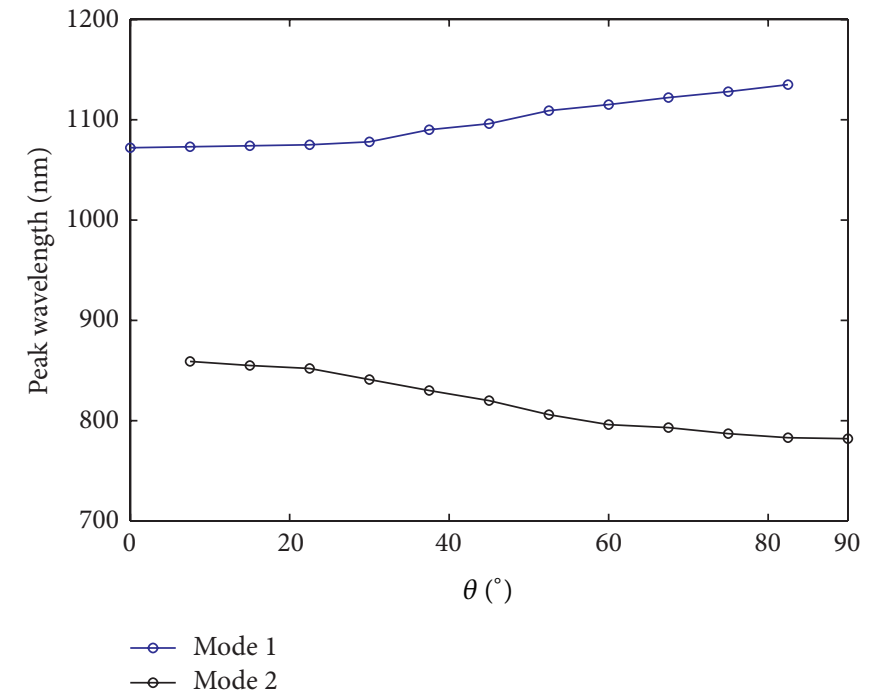

(b)

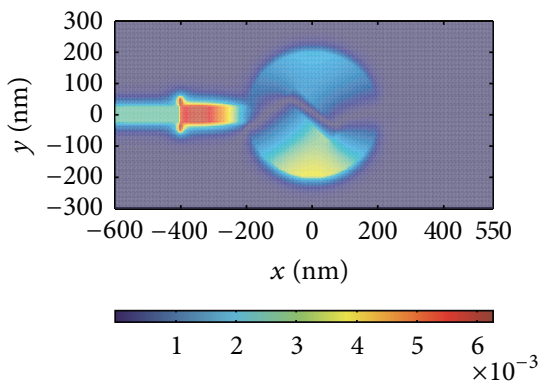

(e)

Figure 5: (a) Transmission response with respect to $\theta=0^{\circ}, 22.5^{\circ}, 45^{\circ}, 67.5^{\circ}$, and $90^{\circ}$. (b) The wavelength at which the transmission spectrum peaks with respect to the block rotation degree. Contour profiles of field $\left|H_{z}\right|$ at (c) $\lambda_{1}=820 \mathrm{~nm},(\mathrm{~d}) \lambda_{2}=1096 \mathrm{~nm}$, and (e) $\lambda_{3}=980 \mathrm{~nm}$ with $\theta=45^{\circ}$.

is $\max$ is $790 \mathrm{~nm}(823 \mathrm{~nm}, 845 \mathrm{~nm}, 903 \mathrm{~nm}$, and $934 \mathrm{~nm})$ for $h=210 \mathrm{~nm}(220 \mathrm{~nm}, 230 \mathrm{~nm}, 240 \mathrm{~nm}$, and $250 \mathrm{~nm})$. Hence, it is clear that the wavelength $\lambda_{C}$ increases monotonously with $h$ increasing as shown in Figure 6(b). That is, the operating wavelength range widens as $h$ decreases. The reason why our structure changes from a filter to a waveguide is that the nanocavity is connected to two MIM waveguides directly, leading to lots of pathways along which SPPs travel. Therefore, the resonant wavelengths differ slightly, which contributes to the change from a filter to a waveguide. For the sake of confirming our analysis, field distributions $\left|H_{z}\right|$ under some resonances are shown in Figures 6(c)-6(e) as examples.

\section{Conclusion}

In this paper, the novel filter effect can be realized based on the MIM plasmonic nanodisk with a metal-block core. The structure without a block inside the cavity is studied to be compared with the proposed structure. The difference between simulation and theoretical results is related to the cavity not being sealed, while (5) is fit for ideal disk. The proposed structure reveals perfect filter effect by easily modulating the height of the block. The resonant wavelengths tend to exhibit a red shift with the increase of the height of the block. The result achieved by FDTD simulations can be accurately analyzed through the EIM and the RMT. We also find the proposed structure produces two modes (i.e., Mode 1 and Mode 2) through tuning the block's rotation degree by means of rotating the strip counterclockwise. Mode 1 and mode 2 tend to exhibit a red shift and a blue shift, respectively. Furthermore, when the width of the block is assumed as $250 \mathrm{~nm}$ and the height of the block changes from $210 \mathrm{~nm}$ to $250 \mathrm{~nm}$, the proposed structure performs as a bend waveguide, not a filter. The FDTD results are verified by the theoretical analysis. Undoubtedly, the ways to give rise to filter effect are ingenious and wonderful. We believe that our fundamental study could find promising applications in the highly integrated plasmonic devices for optical processing and filtering.

\section{Conflict of Interests}

The authors would like to declare that they do not have any commercial or associative interests that represent a conflict of interests in connection with the submitted paper. 

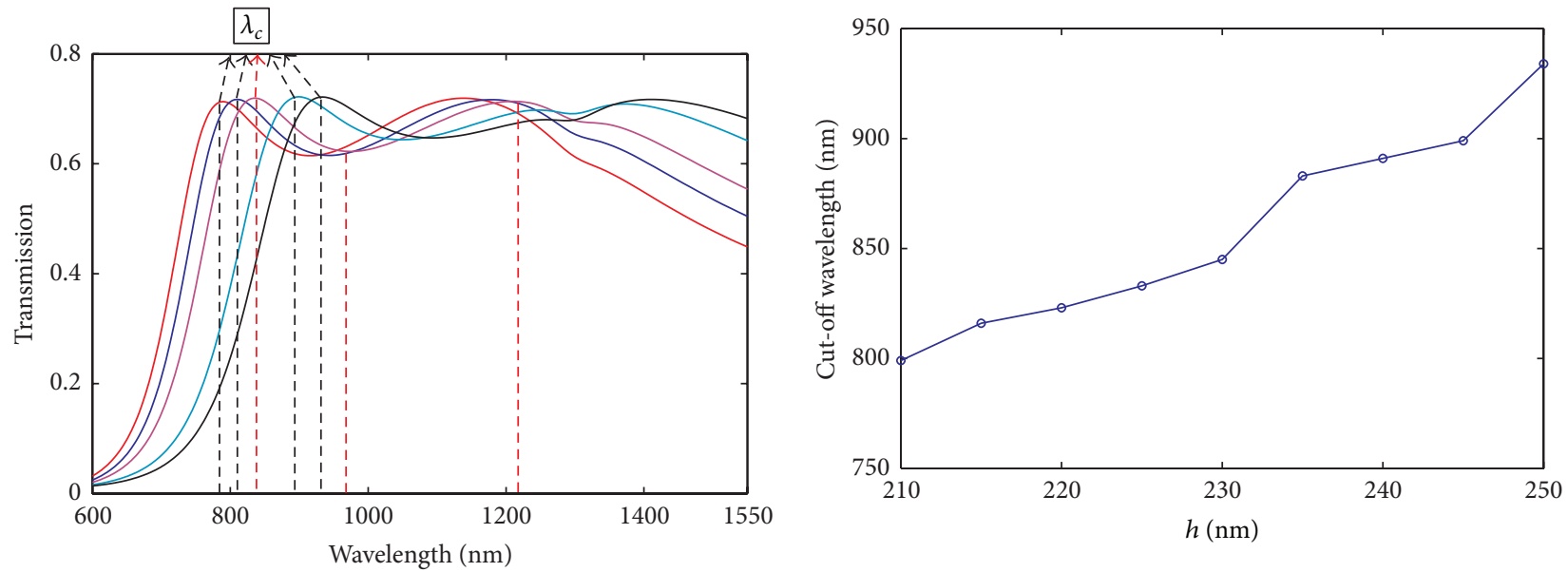

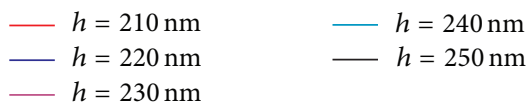

(a)

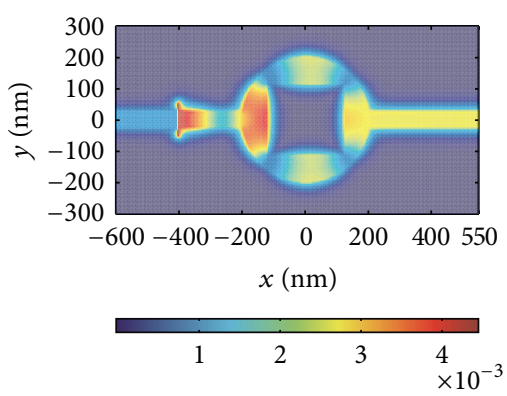

(c)

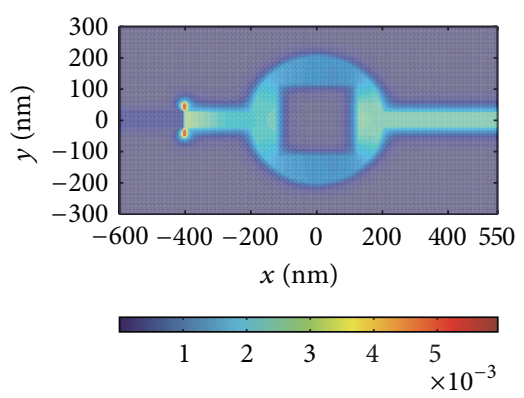

(d) (b)

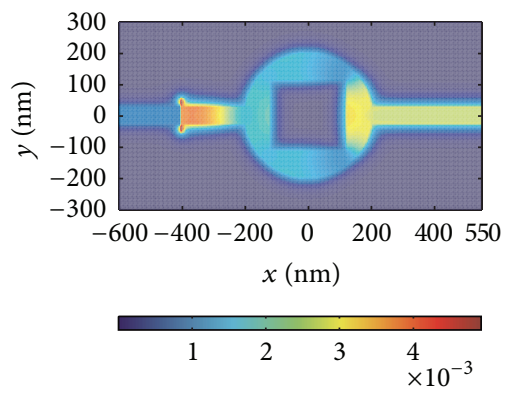

(e)

Figure 6: (a) Transmission response with respect to different heights of the block ( $w=250 \mathrm{~nm}$ ). (b) The cut-off wavelengths with respect to the heights of the block. Contour profiles of field $\left|H_{z}\right|$ at (c) $\lambda=827 \mathrm{~nm}$, (d) $\lambda=1205 \mathrm{~nm}$, and (e) $\lambda=976 \mathrm{~nm}$ (dashed red lines in (a)) with $h=230 \mathrm{~nm}$ and $w=250 \mathrm{~nm}$.

\section{Acknowledgments}

This work was supported by the National Natural Science Foundation of China (Grant nos. 11074069 and 61176116) and the Specialized Research Fund for the Doctoral Program of Higher Education of China (Grant no. 20120161130003).

\section{References}

[1] W. L. Barnes, A. Dereux, and T. W. Ebbesen, "Surface plasmon subwavelength optics," Nature, vol. 424, no. 6950, pp. 824-830, 2003.

[2] S. A. Maier, P. G. Kik, H. A. Atwater et al., "Local detection of electromagnetic energy transport below the diffraction limit in metal nanoparticle plasmon waveguides," Nature Materials, vol. 2, no. 4, pp. 229-232, 2003.

[3] W. H. Weber and G. W. Ford, "Propagation of optical excitations by dipolar interactions in metal nanoparticle chains," Physical Review B-Condensed Matter and Materials Physics, vol. 70, no. 12, 8 pages, 2004.

[4] X.-S. Lin and X.-G. Huang, "Tooth-shaped plasmonic waveguide filters with nanometeric sizes," Optics Letters, vol. 33, no. 23, pp. 2874-2876, 2008.
[5] S. I. Bozhevolnyi, V. S. Volkov, E. Devaux, and T. W. Ebbesen, "Channel plasmon-polariton guiding by subwavelength metal grooves," Physical Review Letters, vol. 95, no. 4, Article ID 046802, 2005.

[6] K. H. Wen, L. S. Yan, W. Pan et al., "Spectral characteristics of plasmonic metal-insulator-metal waveguides with a tilted groove," IEEE Photonics Journal, vol. 4, no. 5, pp. 1794-1800, 2012.

[7] P.-H. Lee and Y.-C. Lan, "Plasmonic waveguide filters based on tunneling and cavity effects," Plasmonics, vol. 5, no. 4, pp. 417$422,2010$.

[8] Y. H. Guo, L. S. Yan, W. Pan et al., "A plasmonic splitter based on slot cavity," Optics Express, vol. 19, no. 15, pp. 13831-13838, 2011.

[9] K. H. Wen, L. S. Yan, W. Pan, B. Luo, Z. Guo, and Y. H. Guo, "A four-port plasmonic quasi-circulator based on metal-insulatormetal waveguides," Optics Express, vol. 20, no. 27, pp. 2802528032, 2012.

[10] F. F. Hu, H. X. Yi, and Z. P. Zhou, "Wavelength demultiplexing structure based on arrayed plasmonic slot cavities," Optics Letters, vol. 36, no. 8, pp. 1500-1502, 2011.

[11] M. A. Bavil, L. Gao, and X. D. Sun, "A compact nanoplasmonics filter and intersection structure based on utilizing a slot cavity 
and a Fabry-Perot Resonator," Plasmonics, vol. 8, no. 2, pp. 631636, 2013.

[12] P. Lalanne, J. P. Hugonin, and J. C. Rodier, "Theory of surface plasmon generation at nanoslit apertures," Physical Review Letters, vol. 95, no. 26, Article ID 263902, 2005.

[13] M. J. Kofke, D. H. Waldeck, Z. Fakhraai, S. Ip, and G. C. Walker, "The effect of periodicity on the extraordinary optical transmission of annular aperture arrays," Applied Physics Letters, vol. 94, no. 2, Article ID 023104, 2009.

[14] T. Nikolajsen, K. Leosson, and S. I. Bozhevolnyi, "Surface plasmon polariton based modulators and switches operating at telecom wavelengths," Applied Physics Letters, vol. 85, no. 24, pp. 5833-5835, 2004.

[15] A. Boltasseva, T. Nikolajsen, K. Leosson, K. Kjaer, M. S. Larsen, and S. I. Bozhevolnyi, "Integrated optical components utilizing long-range surface plasmon polaritons," Journal of Lightwave Technology, vol. 23, no. 1, pp. 413-422, 2005.

[16] P. Berini, "Long-range surface plasmon polaritons," Advances in Optics and Photonics, vol. 1, no. 3, pp. 484-588, 2009.

[17] J. T. Kim, J. J. Ju, S. Park, M.-S. Kim, S. K. Park, and M.-H. Lee, "Chip-to-chip optical interconnect using gold long-range surface plasmon polariton waveguides," Optics Express, vol. 16, no. 17, pp. 13133-13138, 2008.

[18] T. Rosenzveig, P. G. Hermannsson, and K. Leosson, "Modelling of polarization-dependent loss in plasmonic nanowire waveguides," Plasmonics, vol. 5, no. 1, pp. 75-77, 2010.

[19] S. I. Bozhevolnyi, V. S. Volkov, E. Devaux, and T. W. Ebbesen, "Channel plasmon-polariton guiding by subwavelength metal grooves," Physical Review Letters, vol. 95, no. 4, Article ID 046802, 2005.

[20] S. I. Bozhevolnyi, V. S. Volkov, E. Devaux, J. Y. Laluet, and T. W. Ebbesen, "Channel plasmon subwavelength waveguide components including interferometers and ring resonators," Nature, vol. 440, no. 7083, pp. 508-511, 2006.

[21] Z. Y. Fang, H. Qi, C. Wang, and X. Zhu, "Hybrid plasmonic waveguide based on tapered dielectric nanoribbon: excitation and focusing," Plasmonics, vol. 5, no. 2, pp. 207-212, 2010.

[22] J. Park, H. Kim, and B. Lee, "High order plasmonic Bragg reflection in the metal-insulator-metal waveguide Bragg grating," Optics Express, vol. 16, no. 1, pp. 413-425, 2008.

[23] H. Lu, X. M. Liu, D. Mao, L. R. Wang, and Y. K. Gong, "Tunable band-pass plasmonic waveguide filters with nanodisk resonators," Optics Express, vol. 18, no. 17, pp. 17922-17927, 2010.

[24] Z. Zhang, H. Wang, Y. Zhao, and D. Lu, "Transmission properties of the one-end-sealed metal-insulator-metal waveguide," Optik, vol. 124, no. 2, pp. 177-179, 2013.

[25] G. X. Wang and H. Lu, "Unidirectional excitation of surface plasmon polaritons in T-shaped waveguide with nanodisk resonator," Optics Communications, vol. 285, no. 21-22, pp. 41904193, 2012.

[26] K. Wen, L. Yan, W. Pan et al., "Design of plasmonic comb-like filters using loop-based resonators," Plasmonics, vol. 8, no. 2, pp. 1017-1022, 2013.

[27] A. Taflove and S. C. Hagness, Computational Electrodynamics: The Finite-Difference Time-Domain Method, Artech House, Boston, Mass, USA, 2nd edition, 2000.

[28] Z. Han and E. Forsberg, "Surface plasmon bragg gratings formed in metal-insulator-metal waveguides," IEEE Photonics Technology Letters, vol. 19, no. 2, pp. 91-93, 2007.

[29] Q. Zhang, X.-G. Huang, X.-S. Lin, J. Tao, and X.-P. Jin, "A subwavelength coupler-type MIM optical filter," Optics Express, vol. 17, no. 9, pp. 7549-7554, 2009.
[30] S.-L. Qiu and Y.-P. Li, "Q-factor instability and its explanation in the staircased FDTD simulation of high-Q circular cavity," Journal of the Optical Society of America B: Optical Physics, vol. 26, no. 9, pp. 1664-1674, 2009.

[31] I. Chremmos, "Magnetic field integral equation analysis of interaction between a surface plasmon polariton and a circular dielectric cavity embedded in the metal," Journal of the Optical Society of America A, vol. 26, no. 12, pp. 2623-2633, 2009. 

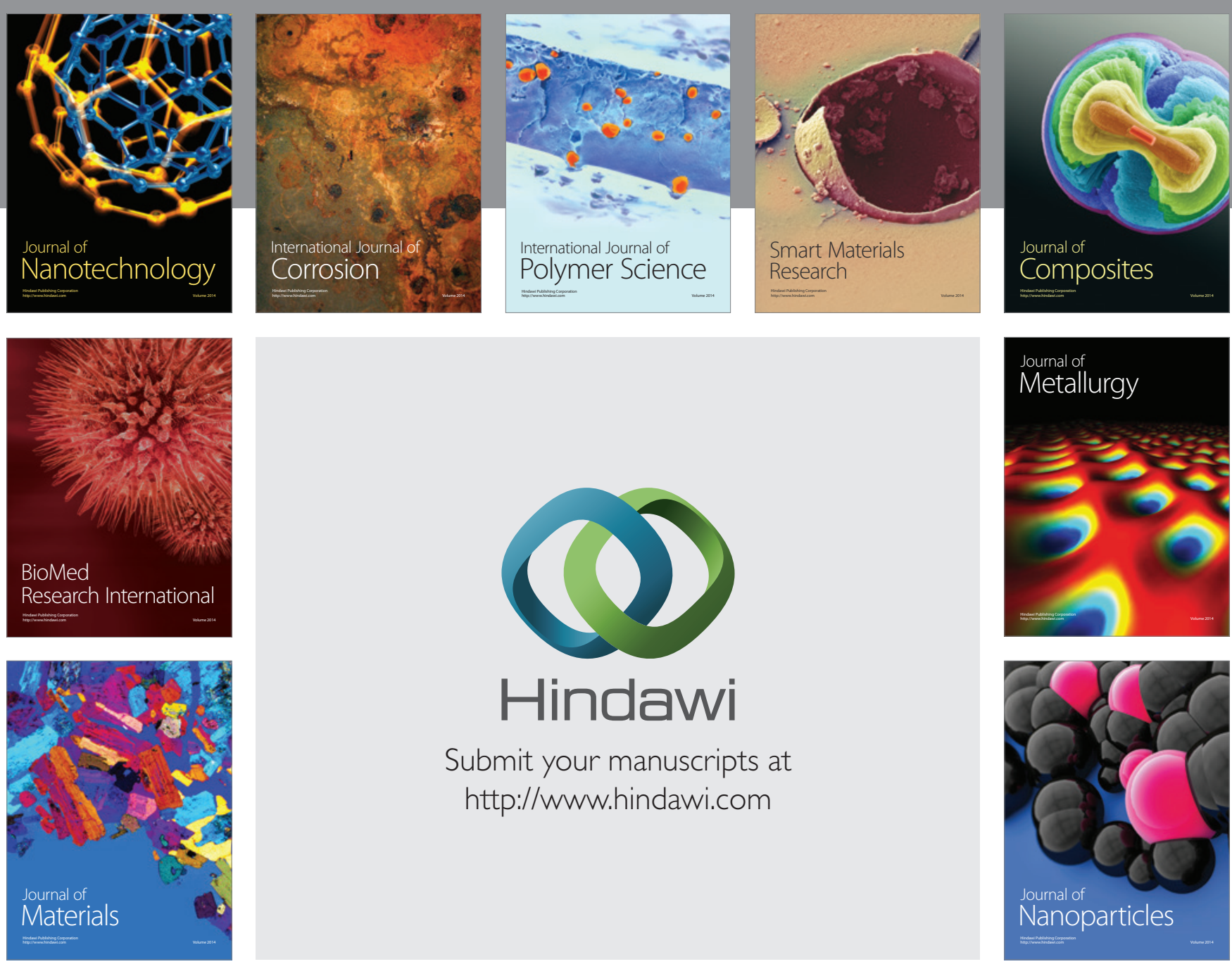

Submit your manuscripts at http://www.hindawi.com
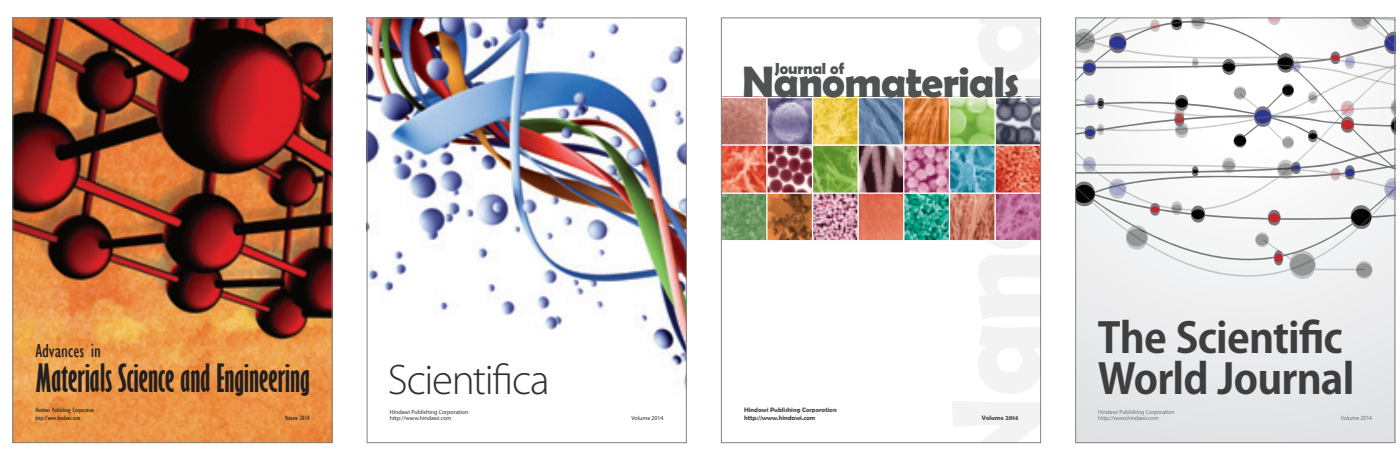

\section{The Scientific World Journal}
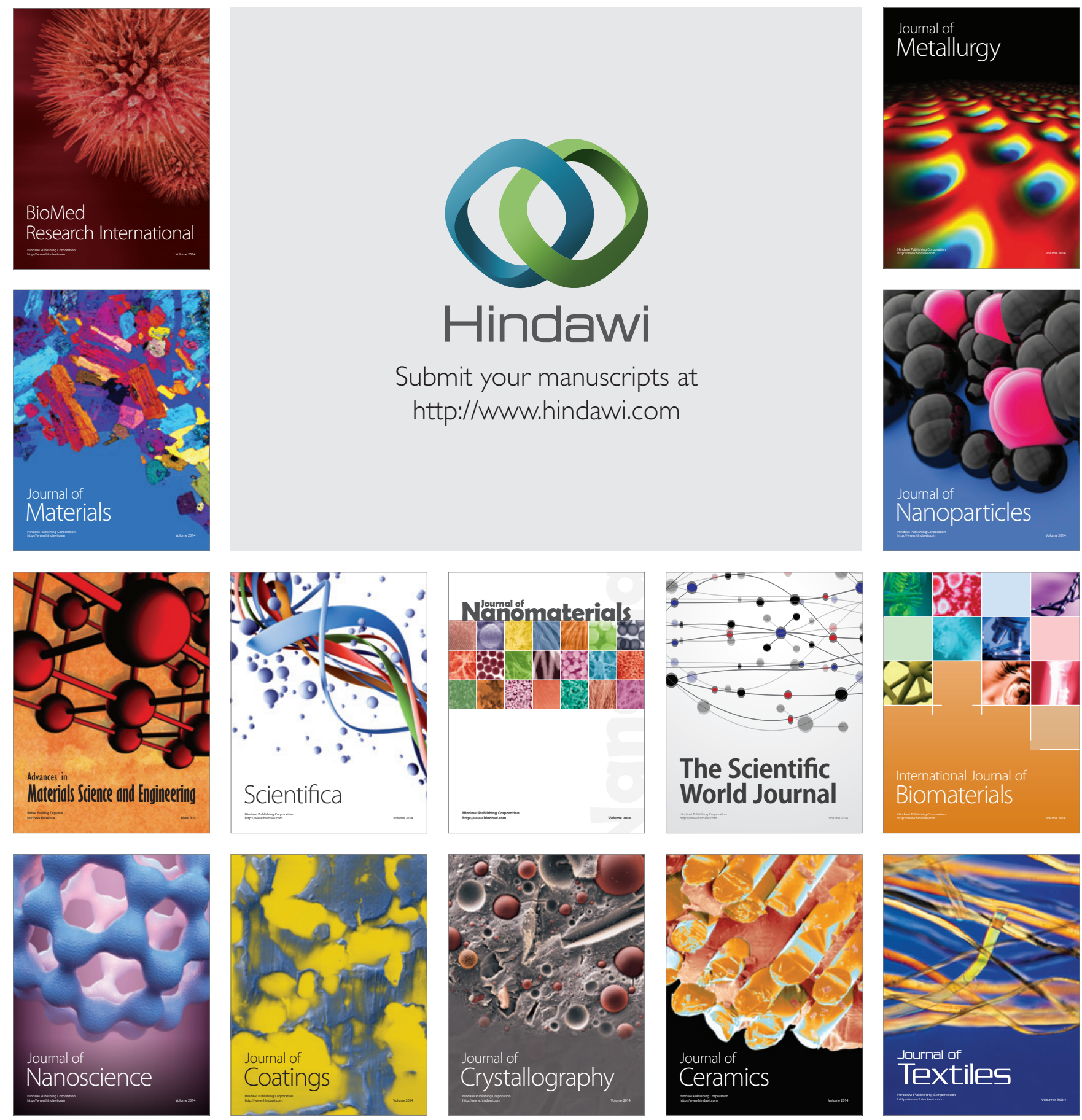